\title{
Comparative microRNA profiling of sporadic and BRCA1 associated basal-like breast cancers
}

Max Yan ${ }^{1 *}$, Kristy Shield-Artin ${ }^{2}$, David Byrne ${ }^{3}$, Siddhartha Deb ${ }^{3}$, Nic Waddell ${ }^{4}$, kConFab Investigators, kConFab, Izhak Haviv ${ }^{2}$ and Stephen B Fox ${ }^{3}$

\begin{abstract}
Background: While a number of studies have examined miRNA profiles across the molecular subtypes of breast cancer, it is unclear whether BRCA1 basal-like cancers have a specific miRNA profile. This study aims to compare grade independent miRNA expression in luminal cancers, sporadic and BRCA1 basal-type breast cancers. It also aims to ascertain an immunohistochemical profile regulated by BRCA1 specific miRNAs for potential diagnostic use.

Methods: miRNA expression was assessed in 11 BRCA1 basal, 16 sporadic basal, 17 luminal grade 3 cancers via microarrays. The expression of Cyclin D1, FOXP1, FIH-1, pan-ERR, NRP1 and CD99, predicted to be regulated by BRCA1 specific miRNAs by computer prediction algorithms, was assessed via immunohistochemistry in a cohort of 35 BRCA1 and 52 sporadic basal-like cancers. Assessment of cyclin D1, FOXP1, NRP1 and CD99 expression was repeated on a validation cohort of 82 BRCA1 and 65 sporadic basal-like breast cancers.

Results: Unsupervised clustering of basal cancers resulted in a "sporadic" cluster of 11 cancers, and a "BRCA1" cluster of 16 cancers, including a subgroup composed entirely of 10 BRCA1 cancers. Compared with sporadic basal cancers, BRCA1 cancers showed reduced positivity for proteins predicted to be regulated by miRNAs: FOXP1 (6/20[30 \%] vs. 37/49[76 \%], $p<0.001)$, cyclin D1 (8/22[36 \%] vs. 30/46[65 \%], $p=0.025)$, NRP1 (2/20[10 \%] vs. 23/46[50 \%], $p=0.002)$. This was confirmed in the validation cohort (all $p<0.001$ ). Negative staining for 2 or more out of FOXP1, cyclin D1 and NRP1 predicts germline BRCA1 mutation with a sensitivity of $92 \%$, specificity of $44 \%$, positive predictive value of $38 \%$ and a negative predictive value of $94 \%$.

Conclusion: Sporadic and BRCA1 basal-like cancers have grade independent miRNA expression profiles. Furthermore miRNA driven differences in the expression of proteins in BRCA1 basal cancers may be detected via immunohistochemistry. These findings may have important diagnostic implications, as immunohistochemical assessment of basal cancers, in addition to the patient's family and clinical history, may potentially identify patients who may benefit from BRCA1 gene testing.
\end{abstract}

Keywords: Breast cancer, microRNA, BRCA1, Basal-like

\section{Background}

Basal-like breast cancers are a subset of breast cancers characterised by triple negativity for ER, PR and HER2, and the expression basal/myoepithelial markers such as CK5/6 [1], CK14 [2] and EGFR [3]. They comprise approximately $15 \%$ of all breast cancers $[1,4]$, and are associated with a more aggressive behaviour and also lack available targeted therapy. It is estimated that $2 \%$ of all

\footnotetext{
* Correspondence: max.yan@sesiahs.health.nsw.gov.au

'Department of Anatomical Pathology, Prince of Wales Hospital, School of Medical Sciences, University of New South Wales, Randwick 2031, Australia Full list of author information is available at the end of the article
}

breast cancers are directly attributable to inherited mutations in the breast cancer susceptibility gene BRCA1 [5]. A strong link exists between BRCA1 mutations and basal phenotype, with $80-90 \%$ of BRCA1 cancers expressing this phenotype [6].

microRNAs are small non-coding RNAs of 20-27 nucleotides that suppress translation through imperfect base pairing with their target mRNAs [7]. It is estimated that $\sim 20 \%$ of mRNA targeted by miRNAs undergo RISC mediated cleavage [8], The remainder may undergo translational silencing without associated changes in mRNA expression [9-11]. Although a number of studies 
examined miRNA profiles across the molecular subtypes of breast cancer [12-16], it is unclear whether the basallike miRNA profiles obtained were independent of tumour proliferation and differentiation as defined by grade. The aim of this study is to: 1 ) derive a basal type miRNA signature that is independent of grade, 2) compare miRNA expression between sporadic and BRCA1 basal cancers to derive a BRCA1 basal signature using formalin fixed paraffin embedded (FFPE) tissue and 3) interrogate via immunohistochemistry the expression of proteins, predicted by computer algorithms, to be regulated by BRCA1 basal specific miRNAs. Identification of a basal type miRNA signature will aid in the targeting of specific miRNAs for further investigation. This is particularly important in view of the limited therapeutic options available for this particularly aggressive variant of breast cancer. It is has been shown that due to their fragment length, miRNA integrity does not appear to be affected storage as archival FFPE tissue [17]. Generation of a "BRCA1 basal" miRNA and immunohistochemical profile in FFPE tissue may identify patients with basal type cancers who will require BRCA1 genetic testing.

\section{Methods}

\section{Samples for miRNA analysis}

Forty-four primary grade III breast cancer (11 BRCA1 basal, 16 sporadic basal, 17 luminal) and 13 normal breast FFPE (formalin fixed, paraffin embedded) specimens were collected for the study. Definitions of intrinsic subtypes were based on ER, HER2 in situ hybridization, EGFR and CK5/6 staining, as per Nielson et al. [3]: Basal cancers (ER negative, HER2 negative, CK5/6 and/or EGFR positive), Luminal cancers (ER positive, HER2 negative). Basal cancers from patients with documented BRCA1 mutations were sourced from kConFab (www.kconfab.org), whereas normal breast tissue, sporadic basal and luminal cancers were collected from the Department of Pathology, Peter MacCallum Cancer Centre and the Victorian Cancer Biobank. Patients with sporadic basal cancers did not have a significant family history as defined by National Cancer Institute guidelines for BRCA1/BRCA2 mutation testing (www.cancer.gov). The clinico-pathological characteristics of the patients included in the study are listed in Additional file 1: Table S1. The study has ethics approval (Peter MacCallum Cancer Centre 09/36). For patients with sporadic cancers, due to the use of archival FFPE tissue, written informed consent was not required by the ethics committee. For BRCA1 patients, written informed consent was obtained as per $\mathrm{kConFab}$ (Kathleen Cuningham Foundation Consortium for research into Familial Breast cancer) biobank guidelines (www.kconfab.org). Three basal (HS578T, MDA-MB-231, MDA-MB-468, all with wild-type BRCA1) and two luminal (MDA-MB-453,
MCF-7) breast cancer cell lines were also included in the study.

\section{RNA extraction}

For primary tumours and normal breast tissue, $10 \mu \mathrm{m}$ thick sections were cut from FFPE tissue blocks. The sections were dewaxed in xylene, placed through $100 \%$ alcohol and allowed to dry. The samples were needle microdissected to ensure the proportion of tumour (or normal epithelium) was greater than $80 \%$, prior to placement into lysis buffer (Agencourt Formapure kit, Beckman Coulter, Beverly, MA, USA). Tissue was digested as per kit protocol (incubate at $70^{\circ} \mathrm{C}$ for $1 \mathrm{~h}$, then add $20 \mu \mathrm{l}$ of Proteinase $\mathrm{K}$ and incubate at $55^{\circ} \mathrm{C}$ for $1 \mathrm{~h}$ ). Total RNA was extracted via a standard TRIZOL(Sigma)/chloroform protocol. For cell lines, total RNA was extracted using the total RNA protocol from the mirVana miRNA Isolation Kit (Ambion, TX, USA). All samples underwent DNase treatment with the Ambion DNA-free kit (Ambion, TX, USA).

\section{miRNA array}

For each sample, $250 \mathrm{ng}$ of total RNA was labelled and hybridized on Human v2 MicroRNA Expression BeadChips (Illumina, San Diego, CA, USA), according to the manufacturers recommendations (Illumina MicroRNA Expression Profiling Assay Guide). The layout of samples across the beadchips is shown in Additional file 1: Table S2. Sixty-nine samples (44 tumour, 13 normal, 7 controls and 5 cell lines) were hybridised on six beadchips across two separate runs: Run 1 ( 1 beadchip, 11 samples) and Run 2 ( 5 beadchips, 58 samples). The sample groups were randomised across the beadchips and also based on position within the beadchip. Controls were included for comparisons between the six beadchips and also between the two separate runs. The correlation of miRNA from control samples across the beadchips are outlined in Additional file 2: Figure S1.

The BeadChips were scanned with the Illumina iScan Reader. Data were imported into GenomeStudio (Illumina), from which raw data with background subtraction were exported to PARTEK Genomics Suite (St. Louis, Missouri, USA) for further analysis. Probes with a maximum intensity value of less than 150 units across all samples were excluded. Of the 1145 probes present on the array, 1037 were used for subsequent analyses. Raw probe intensities were shifted, such that the minimum probe intensity for each sample was equal to 1 . All values were transformed by taking logs (base 2), followed by quantile normalisation [18]. Probe mapping for Illumina MicroRNA Expression v2 BeadChips was based on miRBase v.12.0. 
Differential expression between groups was assessed using ANOVA, with inclusion of the Beadchip number as an independent variable to control for variations between Beadchips. A $p$-value of $<0.05$, after BenjaminiHochberg adjustment for multiple tests, was regarded as significant.

For each miRNA, the expression profiles were standardised to a mean of zero and a standard deviation of 1 prior to unsupervised hierarchical clustering. Clustering was performed using average linkage and Pearson correlation [12]. The full array data is available in GEO (Accession number: GSE61438).

\section{microRNA real time RT-PCR}

Expression of microRNA (miRNA)s hsa-miR-374b, -190b, $-198,-892 \mathrm{a},-130 \mathrm{~b}^{*},-218,-590-3 \mathrm{p}$ and -149 was assayed using real time RT-PCR. cDNA was reversed transcribed from total RNA samples using TaqMan MicroRNA assays and the TaqMan MicroRNA reverse transcription kit (Applied Biosystems). The cDNA was amplified using TaqMan microRNA Assay primers and the TaqMan Universal PCR Mastermix, according to the manufacturer's instructions on the Roche Lightcycler 480. The relative miRNA expression levels were calculated by normalisation with RNU6B expression [19] using the second derivative $(C p)$ method [20]. Comparisons between groups were made using the un-paired $t$-test and correlations with array data were investigated using Pearson correlation on GraphPad Prism 5 (La Jolla, CA, USA).

\section{Prediction of miRNA targets}

Predicted targets of miRNAs were identified via a union search of the two target prediction algorithms miRBase and TargetScan 5.1. Analysis of target protein expression by pSILAC (pulsed stable isotope labelling by amino acids in cell culture) suggests the specificity of these two algorithms are 44 and $61 \%$ respectively [21]. Hence to improve specificity, only genes that are the predicted targets of three or more miRNAs differentially expressed between tumour groups was reported.

Ago2 immunoprecipitation studies by Karginov et al. have shown that approximately $20 \%$ of mRNA targets undergo miRNA-induced cleavage. For the remainder of the targets $(80 \%)$, protein translation is suppressed without changes in mRNA levels [21, 22]. To identify the subset that undergoes miRNA-mediated cleavage, predicted targets derived from above were cross referenced with gene expression array data.

\section{Gene expression array}

Gene expression data for 14 BRCA1 and 10 non-BRCA1 (5 BRCA2 and 5 BRCAX) basal cancers were derived from a cohort previously described by Waddell et al.
(Data available on GEO, accession number GSE19177) [23]. RNA was extracted (Qiagen, Doncaster, VIC) from fresh frozen tissue and gene expression profiling was performed as per the manufacturer's guidelines using $450 \mathrm{ng}$ total RNA and Illumina Human-6 version 2 BeadChips containing 46,000 probes (Illumina Inc., San Diego, CA). Raw data were imported from Illumina Beadstudio v3.2 to PARTEK for further processing. Data were normalized with quantile normalisation, then filtered using an Illumina detection score of $>0.95$ in at least one sample, which yielded 24,004 probes that were used in further analyses.

\section{Immunohistochemistry for miRNA targets}

Tissue microarrays (TMAs) with single $1 \mathrm{~mm}$ cores were constructed. Cyclin D1, FOXP1, FIH-1, pan-ER $\beta$, NRP1 and CD99 immunohistochemistry was performed on TMAs constructed from a cohort of 35 BRCA1 basal cancers from kConFab, and 52 sporadic basal cancers from the Instituti Ospitalieri di Cremona, Italy. Selection of antibodies was based on their previously described associations with BRCA1 status, wherever possible [24-28]. Immunohistochemistry for cyclin D1, FOXP1, NRP1 and CD99 was repeated on a second validation cohort composed of 82 BRCA1 basal cancers from $\mathrm{kConFab}$, and 65 sporadic basal cancers from the Peter MacCallum Cancer Centre, Melbourne. TMA sections were cut from each block at $4 \mu \mathrm{m}$ thick intervals, dewaxed, and placed through graded alcohol and placed into water. The antibody clones used and their titrations are listed in Additional file 1: Table S3. Antigen retrieval, incubation and visualisation for $\mathrm{FIH}$ and pan-ER $\beta$ were performed as per previous published studies [27, 29]. For NRP1 antigen retrieval was performed in a pressure cooker using high $\mathrm{pH}$ EnVision FLEX Target Retrieval Solution (Dako, Glostrup, Denmark) for $2 \mathrm{~min}$. Antigen-antibody complex was detected using Envision FLEX system. FOXP1 and cyclin D1 staining was performed on the Ventana Benchmark ${ }^{\bullet}$ ULTRA system. Antigen retrieval was performed using Ventana ULTRA Cell Conditioner 1 and visualized with Ventana Ultraview Universal DAB. The intensity of staining was scored as negative $=0$; weak staining $=1$; moderate staining $=2$; or strong staining $=3$. The percentage of tumour cells stained in the given core scored as: $0 \%=0$; $1-10 \%=1 ; 11-50 \%=2 ; 51-80 \%=3 ; 81-100 \%=4$. The scores for both staining intensity and the percentage of positive tumour cells were added together to give a maximum score of 7 . Comparisons between groups were based on a chi square (based on presence or absence of staining) and Mann-Whitney U tests (based on scores out of 7) performed on SPSS 16.0 (SPSS, IL, USA). 


\section{Results}

Unsupervised hierarchical cluster analysis reveal distinct microRNA signatures among basal and luminal breast cancers

Unsupervised hierarchical clustering was performed on all 63 samples, based on the expression of 133 miRNAs. Selection of miRNAs was based on the top 75 miRNAs (based on fold change) differentiating between basal and luminal cancers and between BRCA1 and sporadic basal cancers (Fig. 1). To take into account the variation in probe intensity between the two runs (see above), standardisation for each miRNA to a mean of zero and a standard deviation of one was carried out separately for each run.

The analysis accurately separated normal tissue from breast cancer samples. It also separated basal from luminal cancers. There was some overlap (involving a cluster of 4 samples) between normal and luminal cancers. However it is noted that 3 of these 4 samples (normal $\mathrm{N} 8$, N9, luminal cancer L14), were from the smaller run of 11 samples. This overlap may be due to differences in hybridisation between the two different runs, despite an attempt to correct this by standardising the runs separately. Basal cell lines had a different miRNA profile compared to other primary basal cancer samples, and closely resembled the signature for luminal cell lines.
A number of miRNAs correlated with basal phenotype in the current and previous studies. miRNAs that were overexpressed in basal cancers across several studies (following adjustment of $p$ values for multiple tests) include hsa-miR-17/*, 18a/b, 19a, 93, 106a/b, 135b and 142-5p. Similarly hsa-miR-29c/*, 109b, 342-3p/5p, 375 and hsalet-7c were underexpressed in basal cancers (Table 1).

\section{Cluster analysis of basal cancers reveals miRNA signature enriched for BRCA1 cancers}

A cluster analysis based on the top 100 miRNAs discriminating between BRCA1 and sporadic basal cancers was performed on all basal samples (including cell lines). This revealed two distinct signatures among the basal breast cancers (Fig. 2). A "BRCA1" rich cluster of 16 basal cancers, which included all 11 BRCA1 basal cancers plus 5 sporadic basal cancers, and a second "sporadic" basal cluster, composed of the remaining 11 sporadic basal cancers. Within the "BRCA1" cluster there was a subgroup composed entirely of 10 BRCA1 cancers. Basal cell lines, all with wild-type BRCA1, had a profile more closely resembling sporadic basal cancers rather than basal cancers with known BRCA1 mutations. miRNAs that are differentially expressed between BRCA1 and sporadic basal cancers, with a fold change of $>2.5$, are listed in Table 2 .

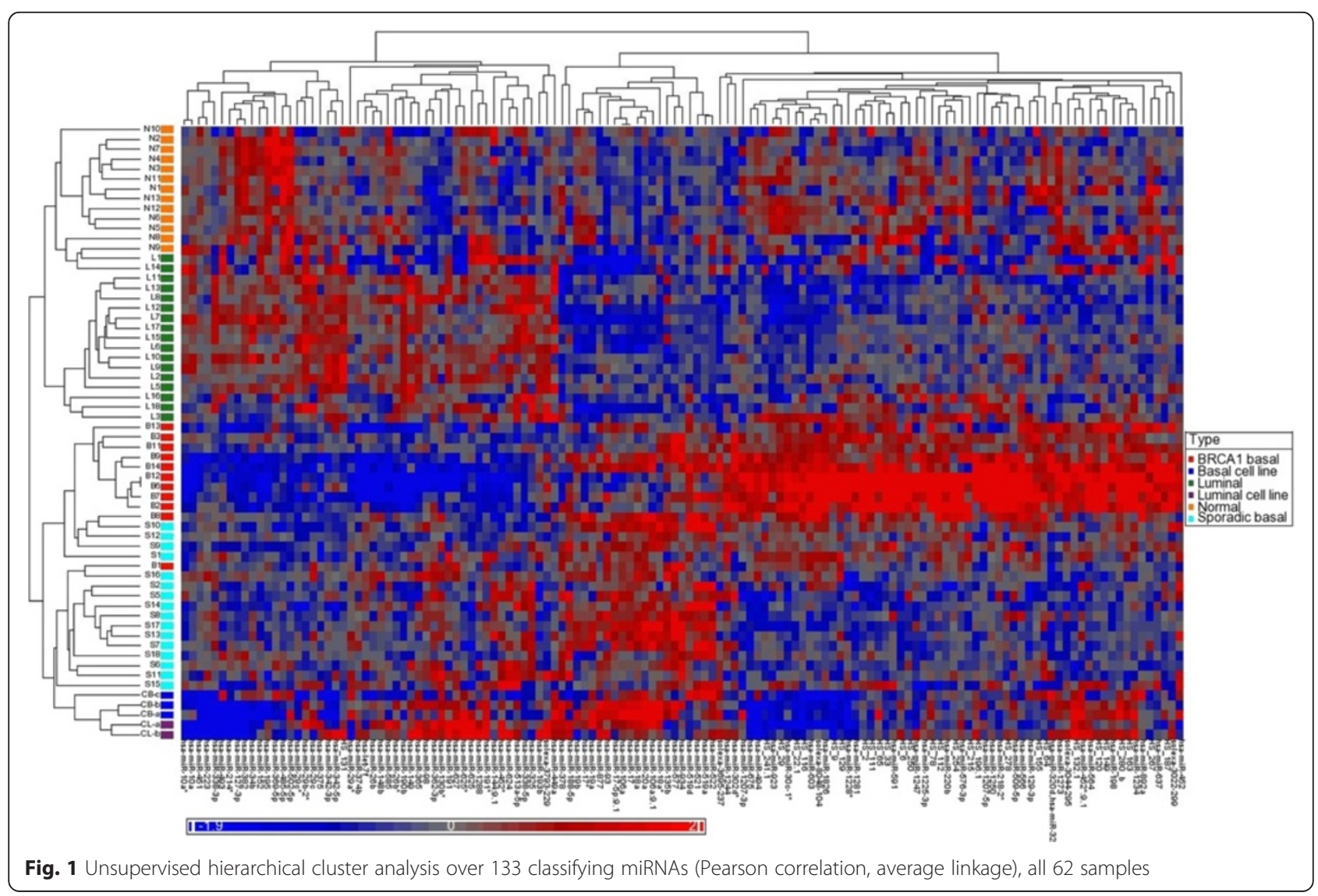


Table 1 Grade independent basal phenotype miRNA signature in common with other studies [12, 13, 49, 50]

\begin{tabular}{|c|c|c|c|c|c|}
\hline & miRNA in common with other studies & Unadjusted $p$ value & Adjusted $p$ value & Fold change & Expression in basal vs. luminal \\
\hline hsa-miR-149 & {$[14,16]$} & 0.0246 & 0.2556 & -2.21 & Down \\
\hline hsa-miR-29c & {$[14-16,50,51]$} & 0.0315 & 0.2838 & -1.22 & Down \\
\hline hsa-miR-29c* & {$[15,16,50]$} & 0.0278 & 0.2679 & -1.61 & Down \\
\hline hsa-miR-109b & {$[14,50]$} & $<0.0001$ & $<0.0001^{*}$ & -11.77 & Down \\
\hline hsa-miR-125a-5p & [14] & 0.0203 & 0.2292 & -1.13 & Down \\
\hline hsa-miR-136 & [12] & 0.0247 & 0.1120 & -1.78 & Down \\
\hline hsa-miR-199a*:9.1 & [12] & 0.0138 & 0.0777 & -1.47 & Down \\
\hline hsa-miR-342-3p & {$[14-16]$} & $0<0.0001$ & $0.0024^{*}$ & -1.58 & Down \\
\hline hsa-miR-342-5p & {$[12-16,49]$} & $<0.0001$ & $0.0001^{*}$ & -1.97 & Down \\
\hline hsa-miR-375 & {$[15,16,50]$} & 0.0005 & 0.0177 & -3.56 & Down \\
\hline hsa-let-7c & {$[12,15]$} & 0.0020 & $0.0232^{*}$ & -1.26 & Down \\
\hline hsa-let-7f & {$[12]$} & 0.0300 & 0.1284 & -1.11 & Down \\
\hline hsa-let-7a & [12] & 0.0467 & 0.1669 & -1.05 & Down \\
\hline hsa-miR-17 & {$[15,16,50]$} & $<0.0001$ & $0.0002^{*}$ & 1.74 & Up \\
\hline hsa-miR-17* & {$[14-16,50]$} & 0.0004 & $0.0162^{*}$ & 1.30 & Up \\
\hline hsa-miR-18a & {$[12-16,32,50,52]$} & $<0.0001$ & $<0.0001^{*}$ & 5.22 & Up \\
\hline hsa-miR-18b & {$[14-16,50]$} & 0.0269 & 0.2676 & 1.89 & Up \\
\hline hsa-miR-19a & {$[14-16,50]$} & 0.0006 & $0.0191^{*}$ & 3.37 & Up \\
\hline hsa-miR-19b & {$[15,16]$} & 0.0005 & $0.0171^{*}$ & 1.90 & Up \\
\hline hsa-miR-93 & {$[12,13,16]$} & 0.0001 & $0.0032^{*}$ & 1.30 & Up \\
\hline hsa-miR-106a & {$[12,13,50]$} & $<0.0001$ & $0.0011^{*}$ & 1.87 & Up \\
\hline hsa-miR-106b & {$[12,13,15,50]$} & 0.0151 & 0.0814 & 1.59 & Up \\
\hline hsa-miR-135b & {$[12-16,50]$} & $<0.0001$ & $0.0017^{*}$ & 4.44 & Up \\
\hline hsa-miR-142-5p & {$[12,13]$} & 0.0019 & $0.0229^{*}$ & 2.07 & Up \\
\hline hsa-miR-20a & {$[15,16]$} & $<0.0001$ & $0.0001^{*}$ & 1.51 & Up \\
\hline hsa-miR-224 & {$[14,16]$} & 0.0004 & 0.0162 & 4.75 & Up \\
\hline hsa-miR-455-5p & [14] & 0.0159 & 0.1986 & 2.08 & Up \\
\hline hsa-miR-505 & [50] & 0.0030 & 0.0741 & 2.83 & Up \\
\hline hsa-miR-519a & [14] & $<0.0001$ & $0.0007^{*}$ & 9.05 & Up \\
\hline hsa-miR-521 & [14] & $<0.0001$ & $<0.0001^{*}$ & 8.97 & Up \\
\hline hsa-miR-522 & [14] & $<0.0001$ & $0.0004^{*}$ & 10.79 & Up \\
\hline hsa-miR-9 & [14] & 0.0069 & 0.1260 & 4.60 & Up \\
\hline hsa-miR-9* & {$[14,16]$} & 0.0322 & 0.2838 & 2.50 & Up \\
\hline hsa-miR-93 & {$[15,16]$} & 0.0003 & 0.0142 & 1.32 & Up \\
\hline
\end{tabular}

*significant $p<0.05$ after adjustment for multiple tests

In order to assess the specificity of the miRNA array signals, seven miRNAs that discriminated between BRCA and sporadic basal cancers in the array were randomly chosen for measurement using reverse transcriptase real-time PCR. Differences between the two groups were validated for four of the seven miRNAs (mir-198, -374b, -218, -149) realtime PCR ( $p=0.0015-0.0289)$ (Additional file 3: Figure S2), yielding a specificity of $57 \%$. For the 4 validated miRNAs, a moderate correlation was seen between data derived from the array and from RT-PCR (Pearson $r=0.503-0.672$, all $p<0.05$ ) (Additional file 4: Figure S3). There was a trend for lower mir-190b expression in BRCA1 cancers, although this was not statistically significant $(p=0.0914)$. No difference in mir-590-3p expression was seen between the two groups $(p=0.962)$. This may be due to reduced accuracy of measurement in the array at low concentrations, as reflected by the low concentrations for these 2 miRNAs $\left(<2^{-6}\right.$ relative to RNU6B) on RT-PCR. The concentration of mir-892a was below detection limit on RTPCR ( $C p>35$ cycles). 


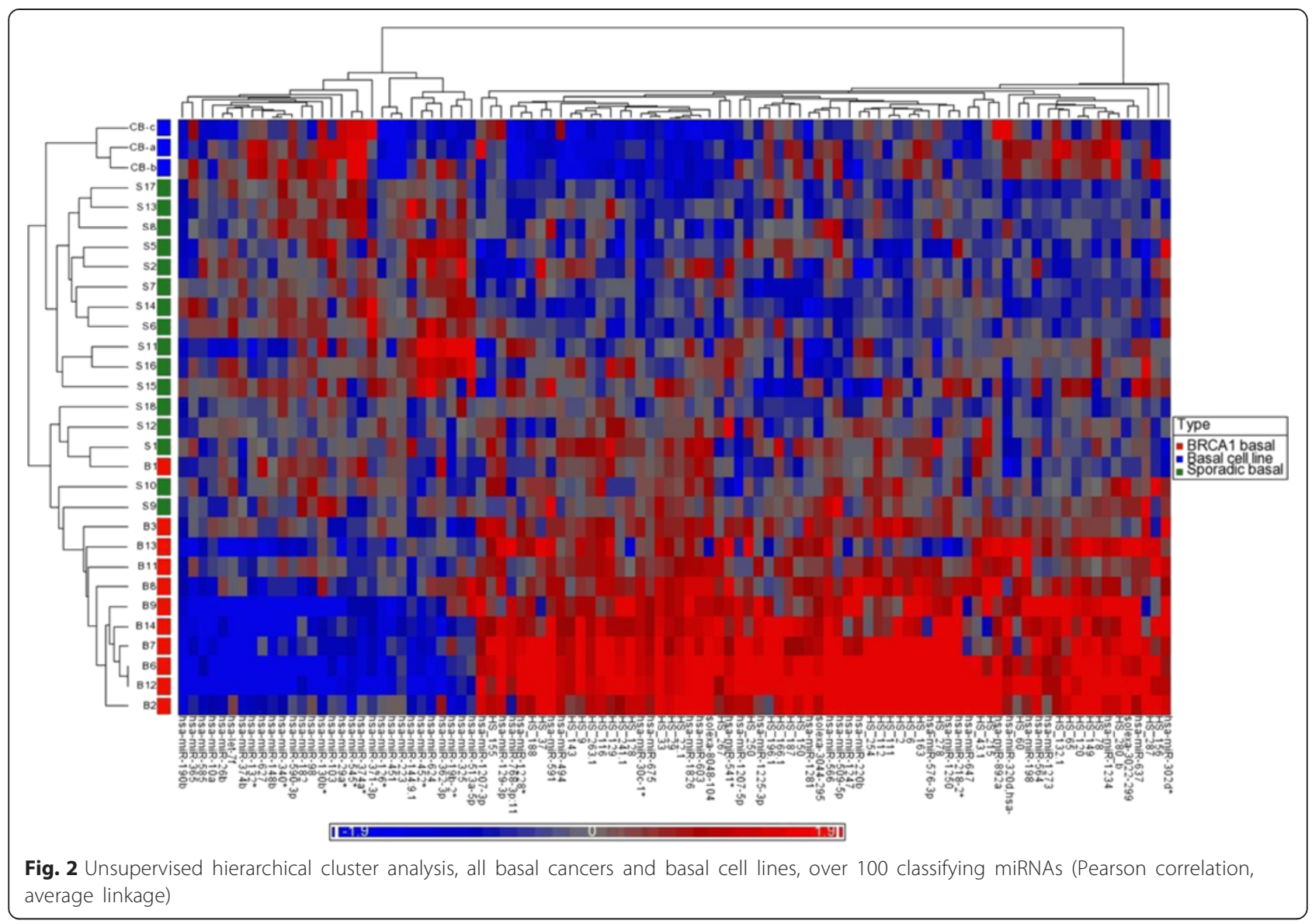

\section{Predicted targets of miRNA differentially expressed between BRCA1 and sporadic basal cancers}

The predicted targets of the top 32 miRNAs differentially expressed between BRCA1 and basal cancers (excluding mir-892, -190b and 590-3p) were sought via a union search of miRBase and TargetScan. Predicted genes targeted by 3 or more miRNAs were retained for a subsequent analysis. This resulted in a list of 1218 genes predicted to be up (562) and down (656) regulated in BRCA1 basal cancers by miRNAs (Additional file 1: Table S4 and S5). Of these 1218 genes, there was overlap of 71 genes $(5.8 \%)$ between the two lists.

The subset of predicted target mRNAs in BRCA1 and sporadic basal cancers, differentially expressed due to miRNA and RISC mediated cleavage was then investigated. Gene expression was compared between 14 BRCA1 basal and 10 non-BRCA1 basal cancers derived from Waddell et al.'s cohort. In a three-dimensional plot generated from an exploratory principle component analysis, the BRCA1 cancers formed a small cluster within a larger cluster incorporating all basal cancers (Fig. 3). Genes differentially expressed between BRCA1 and sporadic basal cancers were cross-matched with list of genes predicted to be targeted by differentially expressed
miRNAs, to obtain a list of genes predicted to be regulated by RISC mediated cleavage (Additional file 1: Table S6).

\section{Immunohistochemical analysis of predicted miRNA} targets in BRCA1 and sporadic basal cancers

The protein expression of four genes predicted to be down-regulated (cyclin D1, FOXP1, NRP1 and ER $\beta$ ) and two predicted to be up-regulated (FIH-1 and CD99) by miRNA expression in BRCA1 vs. sporadic basal cancers were assessed via immunohistochemical staining on TMAs constructed from an cohort of 35 BRCA1 and 52 sporadic basal cancers. BRCA1 basal cancers, when compared to sporadic basal cancers showed reduced positivity for FOXP1 (6/20 [30 \%] vs. 37/49 [76 \%], $p<$ $0.001)$, cyclin D1 (8/22 [36 \%] vs. 30/46 [65 \%], $p=$ 0.025), NRP1 (2/20 [10 \%] vs. 23/46 [50 \%], $p=0.002)$ and CD99 (17/20 [85 \%] vs. 7/19 [37\%], $p=0.002)$ (Table 3, Additional file 5: Figure S4). Differences in expression between the tumour groups were also significant when their scores (out of 7) were compared using a Mann-Whitney $U$ test (all $p \leq 0.005$ ). No differences in FIH-1 and ER $\beta$ expression were seen between the two groups $(p>0.05)$. 
Table 2 miRNAs discriminating BRCA1 from sporadic basal cancers (fold change $>2.5$, adjusted $p<0.05$ )

\begin{tabular}{|c|c|c|c|c|c|}
\hline miRNA & Adjusted $p$ value & Fold change & Expression in BRCA1 vs. basal & Cytoband & Correlation with previous BRCA1 CGH findings \\
\hline hsa-miR-892a & 0.0002 & -7.98 & Up & $\mathrm{Xq27.3}$ & \\
\hline hsa-miR-1247 & 0.0015 & -6.26 & Up & $14 q 32.31$ & \\
\hline hsa-miR-129-3p & 0.0001 & -6.03 & Up & $11 p 11.2$ & \\
\hline hsa-miR-494 & 0.0014 & -5.84 & Up & $14 q 31$ & \\
\hline hsa-miR-1273 & 0.0008 & -5.43 & Up & $8 q 22.2$ & {$[53]$} \\
\hline hsa-miR-198 & 0.0004 & -5.34 & Up & $3 q 13.33$ & [54] \\
\hline hsa-miR-30c- ${ }^{*}$ & $<0.0001$ & -4.51 & Up & $1 \mathrm{p} 34.2$ & \\
\hline hsa-miR-1281 & 0.0001 & -3.88 & Up & $22 q 13.2$ & \\
\hline hsa-miR-220b & $<0.0001$ & -3.76 & Up & 19p13.3 & \\
\hline hsa-miR-566 & $<0.0001$ & -3.75 & Up & $3 p 21.31$ & \\
\hline hsa-miR-603 & 0.0003 & -3.73 & Up & 10p12.1 & {$[53,55]$} \\
\hline hsa-miR-675 & 0.0018 & -3.53 & Up & $11 \mathrm{p} 15.5$ & \\
\hline hsa-miR-637 & 0.0012 & -3.31 & Up & 19p13.3 & \\
\hline hsa-miR-576-3p & 0.0014 & -3.26 & Up & $4 q 25$ & \\
\hline hsa-miR-638 & 0.0366 & -3.04 & Up & 19p13.2 & \\
\hline hsa-miR-1826 & $<0.0001$ & -3.03 & Up & 16p11.2 & {$[54]$} \\
\hline hsa-miR-1268 & 0.0218 & -2.96 & Up & $15 q 11.2$ & {$[53]$} \\
\hline hsa-miR-1234 & 0.0076 & -2.79 & Up & $8 q 24.3$ & {$[53,56]$} \\
\hline hsa-miR-1285 & 0.0428 & -2.69 & Up & $7 q 21.2$ & \\
\hline hsa-miR-509-5p & 0.0043 & -2.53 & Up & Xq27.3 & \\
\hline hsa-miR-374b & 0.0026 & 7.00 & Down & $X q 13.2$ & {$[53]$} \\
\hline hsa-miR-590-3p & 0.0101 & 5.25 & Down & $7 q 11.23$ & \\
\hline hsa-miR-218 & 0.0264 & 4.02 & Down & 4p15.31 & {$[56]$} \\
\hline hsa-miR-335* & 0.0428 & 3.82 & Down & $7 q 32.2$ & \\
\hline hsa-miR-190b & 0.0204 & 3.81 & Down & $1 q 21.3$ & \\
\hline hsa-miR-96 & 0.0405 & 3.48 & Down & $7 q 32.2$ & \\
\hline hsa-miR-454* & 0.0405 & 3.21 & Down & $17 q 22$ & \\
\hline hsa-miR-576-5p & 0.0218 & 3.03 & Down & $4 q 25$ & \\
\hline hsa-miR-340* & 0.0111 & 3.01 & Down & $5 q 35.3$ & {$[53]$} \\
\hline hsa-miR-29a* & 0.0008 & 3.00 & Down & $7 q 32.3$ & \\
\hline hsa-miR-148b & 0.0014 & 2.90 & Down & $17 q 13.13$ & {$[56]$} \\
\hline hsa-miR-130b* & 0.0076 & 2.89 & Down & $22 q 11.21$ & \\
\hline hsa-miR-149 & 0.0443 & 2.77 & Down & $17 q 37.3$ & \\
\hline hsa-miR-10a & 0.0076 & 2.71 & Down & $17 q 21.32$ & \\
\hline hsa-miR-660 & 0.0428 & 2.56 & Down & Xp11.23 & \\
\hline
\end{tabular}

Immunohistochemistry for FOXP1, cyclin D1, NRP1 and CD99 was repeated on a second validation cohort of 82 BRCA1 and 65 sporadic basal cancers. Statistically significant differences were confirmed for FOXP1, cyclin D1 and NRP1 (Pearson chi square all $p<0.001$, Fig. 4 and Table 4). An opposite association for CD99 was observed with sporadic basal cancers showing increased expression compared to BRCA1 cancers $(p=0.024)$. Differences between the tumour groups were also observed when their scores (out of 7) were compared using a Mann-Whitney $U$ test (all $p<$ 0.001).

Using negative staining for 2 or more out of 3 stains (FOXP1, cyclin D1 and NRP1) to predict germline BRCA1 mutation resulted in a sensitivity of $92 \%$ and a specificity of $44 \%$. Assuming 1) the prevalence of BRCA1 in the breast cancer population is $2 \%[5] ; 2$ ) $15 \%$ of breast cancers have a basal phenotype [1, 4]; and 3) $69 \%$ of all BRCA1 cancers have a basal phenotype [30], the positive predictive value (PPV) is 

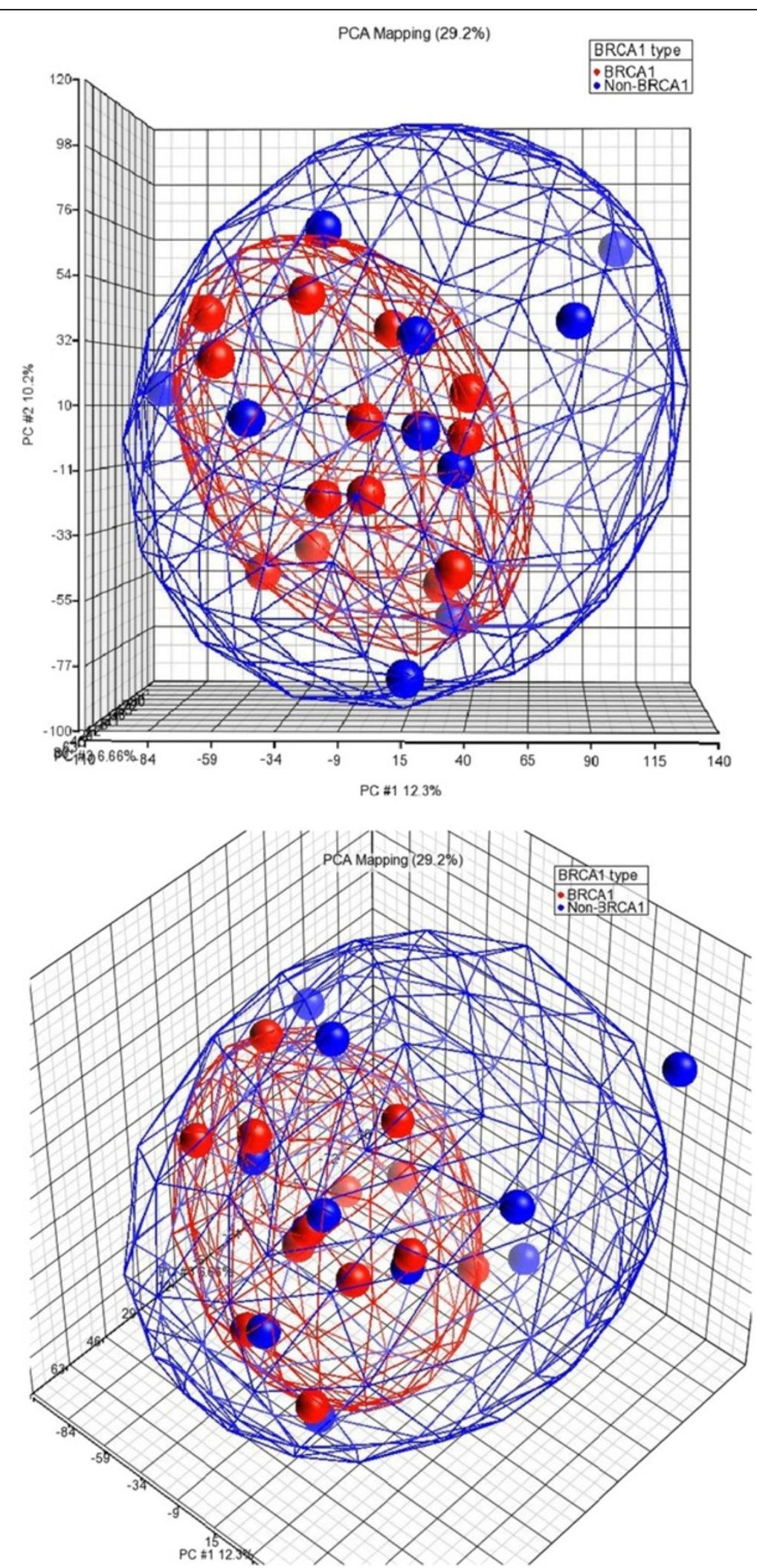

Fig. 3 Three-dimensional PCA plot of gene expression array data for 14 BRCA1 and 10 non-BRCA1 basal cancers 
Table 3 miRNAs and expression of their predicted targets as assessed by immunohistochemistry (IPX) for the initial cohort of 35 BRCA1 and 52 sporadic basal cancers

\begin{tabular}{|c|c|c|c|c|c|c|c|c|c|}
\hline miRNA & $\begin{array}{l}\text { miRNA expression } \\
\text { up or down in } \\
\text { BRCA1 vs. sporadic } \\
\text { cancers }\end{array}$ & $\begin{array}{l}\text { Fold change } \\
\text { miRNA }\end{array}$ & $\begin{array}{l}\text { adjusted } p \text { value } \\
\text { (BRCA1 vs. sporadic) }\end{array}$ & $\begin{array}{l}\text { Predicted target } \\
\text { gene }\end{array}$ & $\begin{array}{l}\text { IPX expression } \\
\text { up or down } \\
\text { in BRCA1 vs. } \\
\text { sporadic cancers }\end{array}$ & $\begin{array}{l}\text { Positive IPX in } \\
\text { BRCA1, } n(\%)\end{array}$ & $\begin{array}{l}\text { Positive IPX in sporadic, } \\
n(\%)\end{array}$ & $\begin{array}{l}p \text { value (chi square) } \\
\text { positive or negative } \\
\text { on IPX }\end{array}$ & $\begin{array}{l}p \text { value (Mann-Whitney) } \\
\text { on IPX score out of } 7\end{array}$ \\
\hline hsa-miR-509-5p & Up & 2.53 & 0.004 & FOXP1 & Down & $6(30 \%)$ & $37(76 \%)$ & $<0.001$ & $<0.001$ \\
\hline hsa-miR-1285 & Up & 2.68 & 0.042 & & & & & & \\
\hline hsa-miR-1826 & Up & 3.02 & $<0.001$ & & & & & & \\
\hline hsa-miR-220b & Up & 3.76 & $<0.001$ & & & & & & \\
\hline hsa-miR-1826 & Up & 3.02 & $<0.001$ & Cyclin D1 & Down & $8(36 \%)$ & $30(65 \%)$ & 0.025 & 0.004 \\
\hline hsa-miR-576-3p & Up & 3.26 & 0.001 & & & & & & \\
\hline hsa-miR-638 & Up & 3.04 & 0.036 & & & & & & \\
\hline hsa-miR-30c-1* & Up & 4.51 & $<0.001$ & NRP1 & Down & $2(10 \%)$ & $23(50 \%)$ & 0.002 & 0.003 \\
\hline hsa-miR-1285 & Up & 2.69 & 0.043 & & & & & & \\
\hline hsa-miR-129-3p & Up & 6.03 & $<0.001$ & & & & & & \\
\hline hsa-miR-29a* & Down & 3.12 & $<0.001$ & CD99 & Up & $17(85 \%)$ & 7 (37 \%) & 0.002 & 0.005 \\
\hline hsa-miR-130b* & Down & 2.95 & $<0.006$ & & & & & & \\
\hline hsa-miR-132* & Down & 2.48 & 0.024 & & & & & & \\
\hline hsa-miR-340* & Down & 3.09 & 0.028 & & & & & & \\
\hline
\end{tabular}




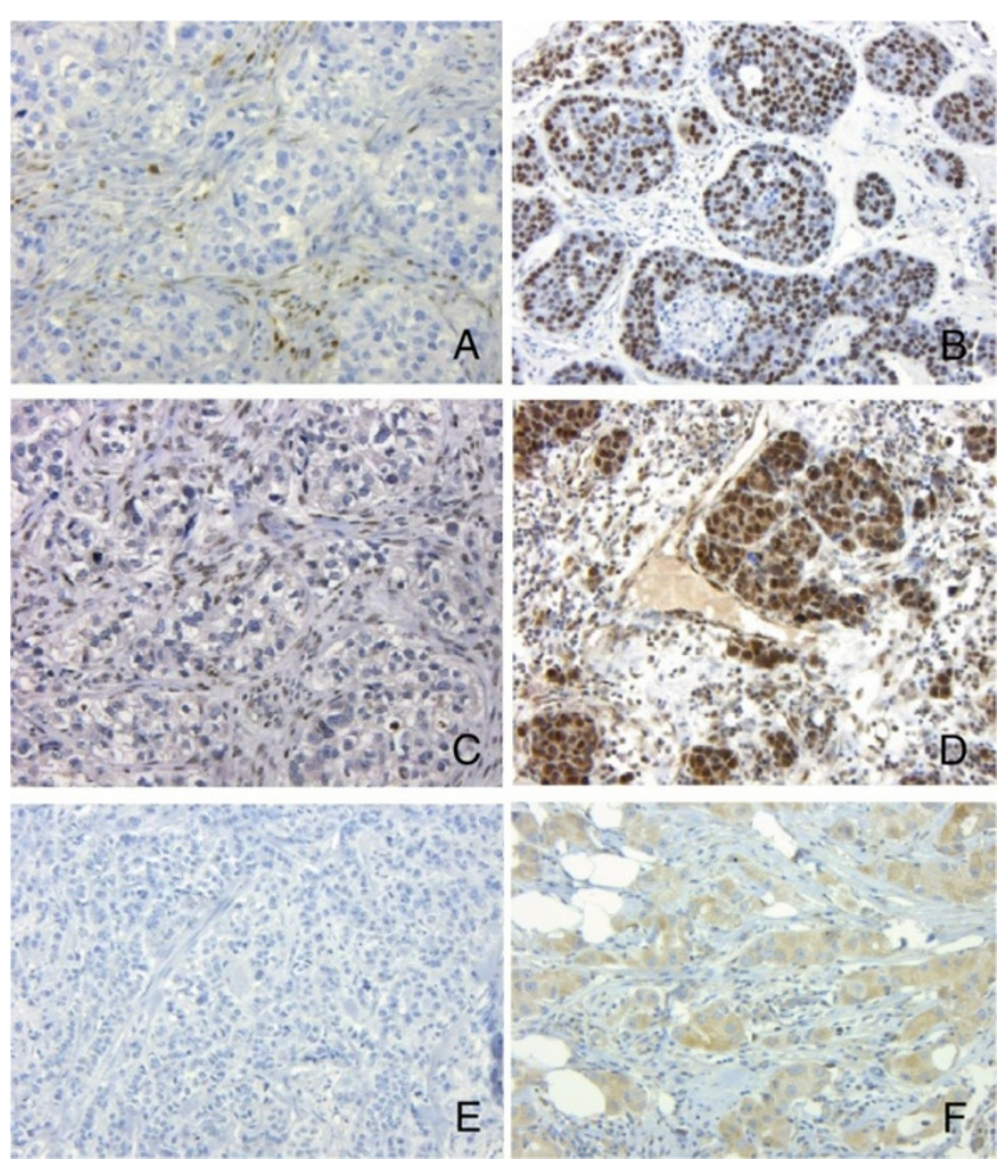

Fig. 4 Negative immunoperoxidase staining for cyclin D1, FOXP1 and NRP1 in BRCA1 basal cancers (a, b and e). Positive staining in sporadic basal cancers for cyclin D1, FOXP1 and NRP1 (b, $\mathbf{d}$ and $\mathbf{f}$ ). Note positive nuclear staining in stromal cells and negative staining in tumour cells for cyclin D1 (a) and FOXP1 (c). (x10, Haematoxylin counterstain)

estimated to be $38 \%$ and the negative predictive value is estimated to be $94 \%$.

\section{Discussion}

Interest in breast cancers with basal features began with Perou et al.'s paper based on cDNA arrays published in 2000 [31]. A number of studies assessing miRNA expression on basal cancers have been published since 2007 but none independent of grade. Thus, it has not been possible to date to reconcile any changes with veracity with regard to subtype or proliferation/differentiation, known drivers of mRNA and miRNA expression [12-16]. The current study, comparing 27 grade 3 basal-like with 17 luminal grade 3 cancers, confirms there are a number of miRNA differentially expressed between luminal and basal cancers, independent of grade, some of which including mir-17-92 cluster (mir-17, 17*, 18a, 19a/b, 20a and 106a), have been linked to basal-like phenotype in these previous studies $[12,14,15,32]$. The expression of this cluster appears to be influenced by DNA copy number [15], and may be involved in the oncogenesis of basallike cancers. These miRNAs promote tumour progression

Table 4 Expression of FOXP1, cyclin D1 and NRP1, as assessed by immunohistochemistry (IPX) for the second validation cohort of 82 BRCA1 and 65 sporadic basal cancers

\begin{tabular}{lllll}
\hline $\begin{array}{l}\text { Predicted target } \\
\text { gene }\end{array}$ & $\begin{array}{l}\text { Positive IPX in BRCA1, } \\
n(\%)\end{array}$ & $\begin{array}{l}\text { Positive IPX in sporadic, } \\
n(\%)\end{array}$ & $\begin{array}{l}p \text { value (chi square) positive } \\
\text { or negative on IPX }\end{array}$ & $\begin{array}{l}p \text { value (Mann-Whitney) on IPX } \\
\text { score out of } 7\end{array}$ \\
\hline FOXP1 & $43(52 \%)$ & $58(94 \%)$ & $<0.001$ & $<0.001$ \\
Cyclin D1 & $47(57 \%)$ & $50(89 \%)$ & $<0.001$ & $<0.001$ \\
NRP1 & $33(41 \%)$ & $42(72 \%)$ & $<0.001$ & $<0.001$ \\
CD99 & $23(28 \%)$ & 0.024 & 0.012 \\
\hline
\end{tabular}


via: reduced stiffness of the extracellular matrix due to reduced expression of PTEN (mir-18a) [32], facilitation of cell migration and metastasis (mir-18b) [33], suppression of tumour suppressor genes ZBTB4 [34] and $\mathrm{Rb}$ (mir-106b) [35]. Loss of miRNAs may also be implicated in the activation of oncogenes, with loss of mir-375 and let-7a being implicated in epithelial-mesenchymal transition in breast cancer cells [36]. Our findings suggest these processes are specific to basal-like cancers, and are independent of tumour differentiation as reflected by the tumour grade.

Unsupervised clustering also suggests that basal-type cancers show significant heterogeneity in miRNA expression. This is in keeping with previous studies by Sotiriou et al. [37] and Kao et al. [38], who divided 26 basal cancers and 13 basal cell lines respectively into 2 subgroups, whereas Kreike et al. [39] divided 97 basal cancers into 5 subgroups. In this study, at one end of the spectrum, there is a subgroup composed entirely of 10 BRCA1 cancers, at the opposite end there is a cluster entirely composed of 11 sporadic cancers, which has a pattern of miRNA expression resembling in vitro basal cell lines. Thus, these data support our findings that BRCA1 cancers have a distinct miRNA signature and form a distinct subgroup within the basal cancers, characterised by the up-regulation of a number of miRNAs involved in regulation of the MAPK/ERK pathway (MPA3K2, MAP2K4, MAP4K4, PTPN2 [40], CHUK [41], PDGFRA [42], ERBB4, JAK3 [43]), and 2) histone modification (HDAC8, MYST2, MLL).

Of the six proteins that were investigated via immunohistochemistry, four (cyclin D1, FOXP1, NRP1 and CD99) showed reduced expression in BRCA1 cancers in the initial cohort with reduced expression for FOXP1, cyclin D1 and NRP1 subsequently confirmed in the validation cohort. Increased expression for CD99 in BRCA1 cancers was not reproduced in the validation cohort, where reduced expression was observed. While there were no accompanying changes in mRNA expression for these proteins, it is known that only $20 \%$ of mRNA targeted by miRNAs undergo RISC mediated cleavage. Hence it is likely that these three genes are part of the majority of genes that undergo translational silencing without an associated reduction in mRNA expression $[21,22]$.

Of these three genes, low cyclin D1 expression has been previously demonstrated in BRCA1 cancers $[24,25]$. Reduced cyclin D1 expression in BRCA1 basal cancers may, in part, be due to the inhibition of translation mediated by mir-576-3p, mir-1826 and mir-638. Conversely, increased cyclin D1 expression in sporadic basal cancers may have important biological implications. For instance, direct targeting of cyclin D1 by NOTCH1 and 3 has been linked to cell cycle progression in basal cancers [44]. In addition, phosphorylation of BRCA1 by cyclin D1 has been shown to interfere with DNA dependent activities of BRCA1 [45]. Expression of NRP1 in sporadic cancers may have potentially important therapeutic applications, in view of the development of anti-NRP monoclonal antibodies and cell penetrating peptides [46].

Using negative staining for 2 or more out of 3 stains (FOXP1, cyclin D1 and NRP1) to predict germline BRCA1 mutation resulted in a positive predictive value (PPV) of $38 \%$ and a negative predictive value of $94 \%$. While this PPV is relatively low due to the rarity of BRCA1 mutations in the population, its value is likely to improve in patients with a family history of breast cancer and/or early onset cancers. Nevertheless, our findings suggest immunohistochemistry for FOXP1/NRP1/ cyclin D1 may be useful, in conjunction with family history, in selecting patients with basal cancers for BRCA1 screening.

\section{Conclusion}

In summary, our study demonstrates basal-like cancers have a grade independent miRNA expression profile. Furthermore miRNA driven differences in the expression of proteins by BRCA1 vs. sporadic basal cancers may be detected via immunohistochemical staining of paraffin embedded tissue. These findings may have important diagnostic implications, as immunohistochemical assessment of basal cancers, in addition to the patient's family and clinical history, may potentially identify patients who may benefit from BRCA1 gene testing. Lastly, there is evidence to suggest BRCA1 deficient cancers may be sensitive to PARP inhibitors [47]. Hence stratification of basal-like cancers, based on the "BRCA1-ness" of their miRNA signature, generated from archival FFPE tissue, may be highly relevant to clinical trials investigating targeted therapies, such as PARP inhibitors [48]. Validation of these findings in larger patient cohorts, however, will be required to assess the diagnostic utility of such an approach.

\section{Additional files}

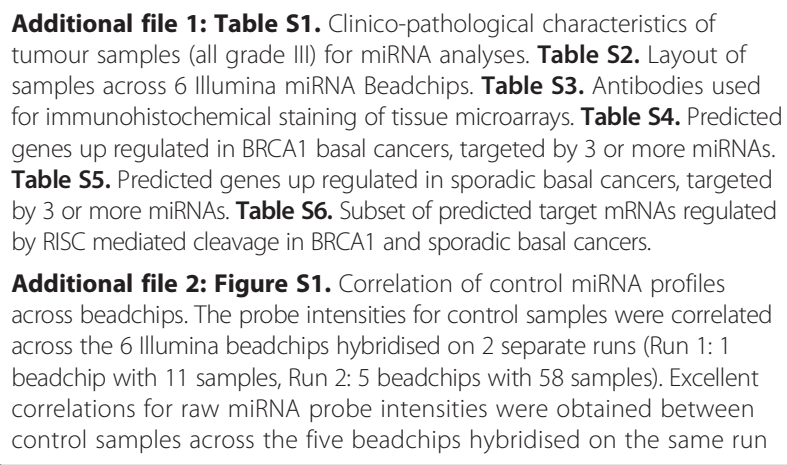

Additional file 1: Table S1. Clinico-pathological characteristics of tumour samples (all grade III) for miRNA analyses. Table S2. Layout of samples across 6 Illumina miRNA Beadchips. Table S3. Antibodies used for immunohistochemical staining of tissue microarrays. Table S4. Predicted genes up regulated in BRCA1 basal cancers, targeted by 3 or more miRNAs. Table S5. Predicted genes up regulated in sporadic basal cancers, targeted by 3 or more miRNAs. Table S6. Subset of predicted target mRNAs regulated by RISC mediated cleavage in BRCA1 and sporadic basal cancers.

Additional file 2: Figure S1. Correlation of control miRNA profiles across beadchips. The probe intensities for control samples were correlated across the 6 Illumina beadchips hybridised on 2 separate runs (Run 1: 1 beadchip with 11 samples, Run 2: 5 beadchips with 58 samples). Excellent correlations for raw miRNA probe intensities were obtained between control samples across the five beadchips hybridised on the same run 
(Run 2, Spearman $r=0.9631-0.9906$, all $p<0.001$ ) (Additional file 2: Figure S1a). For control samples hybridised on different runs, a weaker correlation was observed (Spearman $r=0.8139, p<0.001$ ) (Additional file 2: Figure S1b). Occasional probes were observed to have a much stronger signal in the smaller run 1 (4687786010_L) compared to run 2 (4726968002_E). A small improvement in the correlation between these two samples was observed following quantile normalisation and log 2 transformation (Pearson $r=0.8538, p<0.001$ ) (Additional file 2: Figure S1c). Additional file 2: Figure S1a. Scatterplot of raw miRNA probe intensities from control samples across 2 beadchips hybridised on the same run (Spearman $r=0.9906, p<0.001$ ); Additional file 2: Figure S1b. Scatterplot of raw miRNA probe intensities from control samples hybridised on separate runs (Spearman $r=0.8139, p<0.001$ ); Additional file 2: Figure S1c. Scatterplot of probe intensities of control samples (from Additional file 2: Figure S1b), hybridised on separate runs following quantile normalisation and $\log 2$ transformation (Pearson $r=0.8538, p<0.001$ ).

Additional file 3: Figure S2. RT-PCR validation of mir-374b, mir-149, mir-218 and mir-198 in discriminating between BRCA1 and sporadic basal breast cancers (all $p<0.05$ ). A trend was seen for mir-190b although this was not statistically significant $(p=0.0914)$. No differences in the expression of mir-590-3p were observed ( $p=0.9623)$.

Additional file 4: Figure S3. Correlation between microarray and RT-PCR (relative to RNU6B) measurements for mir-198 $(r=0.672, p=0.0023)$, mir-149 $(r=0.567, p=0.0344)$, mir-218 $(r=0.521, p=0.0385)$ and mir-374b $(r=0.503$, $p=0.0335$.

Additional file 5: Figure S4. Distribution of scores in BRCA1 and sporadic basal type cancers for cyclin D1 $(p=0.004)$, FOXP1 $(p<0.001)$ and NRP1 $(p=0.003)$.

\section{Competing interests}

The authors declare that they have no competing interests.

\section{Authors' contributions}

MY contributed to the conception and design of the study, analyzed the data, and drafted and revised the manuscript. KS performed the microRNA arrays. DB and SD performed and analysed the immunohistochemistry studies, NW performed the CGH and mRNA array experiments. $1 \mathrm{H}$ contributed to the statistical analysis and design of the miRNA array experiments. kConFab contributed to the provision of study materials and the collection of clinicopathologic and follow-up survival data. SBF contributed to the conception and design of the study, provision of study materials, analysis, drafting, and revision of the manuscript. All authors read and approved the final manuscript.

\section{Authors' information}

kConFab Investigators, kConFab, Peter MacCallum Cancer Centre, East Melbourne, 3002, Australia, email: heather.thorne@petermac.org.

\section{Acknowledgements}

We thank Heather Thorne, Eveline Niedermayr, the kConFab research nurses and staff, the staff of the Family Cancer Clinics, and the Clinical Follow Up Study (funded by NHMRC grants 145684, 288704, and 454508) for the collection of data and tissue specimens. kConFab is supported by grants from the National Breast Cancer Foundation, the National Health and Medical Research Council (NHMRC), and by the Queensland Cancer Fund, the Cancer Councils of New South Wales, Victoria, Tasmania, and South Australia, and the Cancer Foundation of Western Australia. This study was partly funded by the Victorian Breast Cancer Research Consortium, the NHMRC, the Cancer Council Victoria and the Victorian Cancer Biobank (MY, SD, SF).

\section{Author details}

1Department of Anatomical Pathology, Prince of Wales Hospital, School of Medical Sciences, University of New South Wales, Randwick 2031, Australia. ${ }^{2}$ Baker IDI Heart and Diabetes Institute, Prahran 3004, Australia. ${ }^{3}$ Department of Pathology, Peter MacCallum Cancer Centre, East Melbourne 3002, Australia. ${ }^{4}$ Queensland Centre for Medical Genomics, Institute for Molecular Bioscience, University of Queensland, St Lucia, Australia.
Received: 18 November 2014 Accepted: 26 June 2015

Published online: 08 July 2015

\section{References}

1. van de Rijn M, Perou CM, Tibshirani R, Haas P, Kallioniemi O, Kononen J, et al. Expression of cytokeratins 17 and 5 identifies a group of breast carcinomas with poor clinical outcome. Am J Pathol. 2002;161(6):1991-6.

2. Fulford LG, Reis-Filho JS, Ryder K, Jones C, Gillett CE, Hanby A, et al. Basallike grade III invasive ductal carcinoma of the breast: patterns of metastasis and long-term survival. Breast Cancer Res. 2007;9(1):R4.

3. Nielsen TO, Hsu FD, Jensen K, Cheang M, Karaca G, Hu Z, et al. Immunohistochemical and clinical characterization of the basal-like subtype of invasive breast carcinoma. Clin Cancer Res. 2004;10(16):5367-74.

4. Rodriguez-Pinilla SM, Sarrio D, Honrado E, Hardisson D, Calero F, Benitez J, et al. Prognostic significance of basal-like phenotype and fascin expression in node-negative invasive breast carcinomas. Clin Cancer Res. 2006;12(5):1533-9.

5. Lakhani SR, Reis-Filho JS, Fulford L, Penault-Llorca F, van der Vijver M, Parry $\mathrm{S}$, et al. Prediction of BRCA1 status in patients with breast cancer using estrogen receptor and basal phenotype. Clin Cancer Res. 2005;11(14):5175-80.

6. Foulkes WD, Stefansson IM, Chappuis PO, Begin LR, Goffin JR, Wong N, et al. Germline BRCA1 mutations and a basal epithelial phenotype in breast cancer. J Natl Cancer Inst. 2003;95(19):1482-5.

7. Bartel DP. MicroRNAs: genomics, biogenesis, mechanism, and function. Cell. 2004;116(2):281-97.

8. Hutvagner G, Zamore PD. A microRNA in a multiple-turnover RNAi enzyme complex. Science. 2002;297(5589):2056-60.

9. Engels BM, Hutvagner G. Principles and effects of microRNA-mediated posttranscriptional gene regulation. Oncogene. 2006;25(46):6163-9.

10. Olsen PH, Ambros V. The lin-4 regulatory RNA controls developmental timing in Caenorhabditis elegans by blocking LIN-14 protein synthesis after the initiation of translation. Dev Biol. 1999;216(2):671-80.

11. Petersen CP, Bordeleau ME, Pelletier J, Sharp PA. Short RNAs repress translation after initiation in mammalian cells. Mol Cell. 2006;21(4):533-42.

12. Blenkiron C, Goldstein LD, Thorne NP, Spiteri I, Chin SF, Dunning MJ, et al. MicroRNA expression profiling of human breast cancer identifies new markers of tumor subtype. Genome Biol. 2007:8(10):R214.

13. Janssen EA, Slewa A, Gudlaugsson E, Jonsdottir K, Skaland I, Soiland H, et al. Biologic profiling of lymph node negative breast cancers by means of microRNA expression. Mod Pathol. 2010;23(12):1567-76.

14. Enerly E, Steinfeld I, Kleivi K, Leivonen SK, Aure MR, Russnes HG, et al. miRNA-mRNA integrated analysis reveals roles for miRNAs in primary breast tumors. PLoS One. 2011;6(2):e16915.

15. de Rinaldis E, Gazinska P, Mera A, Modrusan Z, Fedorowicz GM, Burford B, et al. Integrated genomic analysis of triple-negative breast cancers reveals novel microRNAs associated with clinical and molecular phenotypes and sheds light on the pathways they control. BMC Genomics. 2013;14:643.

16. Dvinge H, Git A, Graf S, Salmon-Divon M, Curtis C, Sottoriva A, et al. The shaping and functional consequences of the microRNA landscape in breast cancer. Nature. 2013:497(7449):378-82.

17. Siebolts U, Varnholt H, Drebber U, Dienes HP, Wickenhauser C, Odenthal M Tissues from routine pathology archives are suitable for microRNA analyses by quantitative PCR. J Clin Pathol. 2009;62(1):84-8.

18. Pradervand S, Weber J, Thomas J, Bueno M, Wirapati P, Lefort K, et al. Impact of normalization on miRNA microarray expression profiling. RNA. 2009;15(3):493-501.

19. Tavazoie SF, Alarcon C, Oskarsson T, Padua D, Wang Q, Bos PD, et al. Endogenous human microRNAs that suppress breast cancer metastasis. Nature. 2008:451(7175):147-52.

20. Luu-The V, Paquet N, Calvo E, Cumps J. Improved real-time RT-PCR method for high-throughput measurements using second derivative calculation and double correction. Biotechniques. 2005;38(2):287-93.

21. Selbach M, Schwanhausser B, Thierfelder N, Fang Z, Khanin R, Rajewsky N. Widespread changes in protein synthesis induced by microRNAs. Nature. 2008;455(7209):58-63.

22. Karginov FV, Conaco C, Xuan Z, Schmidt BH, Parker JS, Mandel G, et al. A biochemical approach to identifying microRNA targets. Proc Natl Acad Sci U S A. 2007;104(49):19291-6.

23. Waddell N, Arnold J, Cocciardi S, da Silva L, Marsh A, Riley J, et al. Subtypes of familial breast tumours revealed by expression and copy number profiling. Breast Cancer Res Treat. 2010;123(3):661-77. 
24. Aaltonen $\mathrm{K}$, Blomqvist $\mathrm{C}$, Amini RM, Eerola H, Aittomaki K, Heikkila P, et al. Familial breast cancers without mutations in BRCA1 or BRCA2 have low cyclin E and high cyclin D1 in contrast to cancers in BRCA mutation carriers. Clin Cancer Res. 2008;14(7):1976-83.

25. Armes JE, Trute L, White D, Southey MC, Hammet F, Tesoriero A, et al. Distinct molecular pathogeneses of early-onset breast cancers in BRCA1 and BRCA2 mutation carriers: a population-based study. Cancer Res. 1999:59(8):2011-7.

26. Rayoo M, Yan M, Takano EA, Bates GJ, Brown PJ, Banham AH, et al. Expression of the forkhead box transcription factor FOXP1 is associated with oestrogen receptor alpha, oestrogen receptor beta and improved survival in familial breast cancers. J Clin Pathol. 2009;62(10):896-902

27. Yan M, Rayoo M, Takano EA, Fox SB. Nuclear and cytoplasmic expressions of ERbeta1 and ERbeta2 are predictive of response to therapy and alters prognosis in familial breast cancers. Breast Cancer Res Treat. 2011;126(2):395-405.

28. Yan M, Rayoo M, Takano EA, Investigators KC, Fox SB. BRCA1 tumours correlate with a HIF-1alpha phenotype and have a poor prognosis through modulation of hydroxylase enzyme profile expression. Br J Cancer. 2009;101(7):1168-74

29. Tan EY, Yan M, Campo L, Han C, Takano E, Turley H, et al. The key hypoxia regulated gene CAIX is upregulated in basal-like breast tumours and is associated with resistance to chemotherapy. Br J Cancer. 2009;100(2):405-11.

30. Mavaddat N, Barrowdale D, Andrulis IL, Domchek SM, Eccles D, Nevanlinna $\mathrm{H}$, et al. Pathology of breast and ovarian cancers among BRCA1 and BRCA2 mutation carriers: results from the Consortium of Investigators of Modifiers of BRCA1/2 (CIMBA). Cancer Epidemiol Biomark Prev. 2012;21(1):134-47.

31. Perou CM, Sorlie T, Eisen MB, van de Rijn M, Jeffrey SS, Rees CA, et al. Molecular portraits of human breast tumours. Nature. 2000;406(6797):747-52.

32. Mouw JK, Yui Y, Damiano L, Bainer RO, Lakins JN, Acerbi I, et al. Tissue mechanics modulate microRNA-dependent PTEN expression to regulate malignant progression. Nat Med. 2014;20(4):360-7.

33. Fonseca-Sanchez MA, Perez-Plasencia C, Fernandez-Retana J, ArechagaOcampo E, Marchat LA, Rodriguez-Cuevas S, et al. microRNA-18b is upregulated in breast cancer and modulates genes involved in cell migration. Oncol Rep. 2013;30(5):2399-410.

34. Kim K, Chadalapaka G, Lee SO, Yamada D, Sastre-Garau X, Defossez PA, et al. Identification of oncogenic microRNA-17-92/ZBTB4/specificity protein axis in breast cancer. Oncogene. 2012;31(8):1034-44.

35. Gong C, Qu S, Liu B, Pan S, Jiao Y, Nie Y, et al. MiR-106b expression determines the proliferation paradox of TGF-beta in breast cancer cells. Oncogene. 2015;34(1):84-93

36. Liu Y, Li H, Feng J, Cui X, Huang W, Li Y, et al. Lin28 induces epithelial-tomesenchymal transition and stemness via downregulation of let-7a in breast cancer cells. PLoS One. 2013;8(12):e83083.

37. Sotiriou C, Wirapati P, Loi S, Harris A, Fox S, Smeds J, et al. Gene expression profiling in breast cancer: understanding the molecular basis of histologic grade to improve prognosis. J Natl Cancer Inst. 2006;98(4):262-72.

38. Kao J, Salari K, Bocanegra M, Choi YL, Girard L, Gandhi J, et al. Molecular profiling of breast cancer cell lines defines relevant tumor models and provides a resource for cancer gene discovery. PLoS One. 2009;4(7):e6146.

39. Kreike $B$, van Kouwenhove $M$, Horlings $H$, Weigelt $B$, Peterse $H$, Bartelink $H$, et al. Gene expression profiling and histopathological characterization of triple-negative/basal-like breast carcinomas. Breast Cancer Res. 2007;9(5):R65.

40. van Vliet C, Bukczynska PE, Puryer MA, Sadek CM, Shields BJ, Tremblay ML, et al. Selective regulation of tumor necrosis factor-induced Erk signaling by Src family kinases and the T cell protein tyrosine phosphatase. Nat Immunol. 2005:6(3):253-60

41. Takada Y, Kobayashi Y, Aggarwal BB. Evodiamine abolishes constitutive and inducible NF-kappaB activation by inhibiting IkappaBalpha kinase activation, thereby suppressing NF-kappaB-regulated antiapoptotic and metastatic gene expression, up-regulating apoptosis, and inhibiting invasion. J Biol Chem. 2005;280(17):17203-12.

42. Jurek A, Amagasaki K, Gembarska A, Heldin CH, Lennartsson J. Negative and positive regulation of MAPK phosphatase 3 controls platelet-derived growth factor-induced Erk activation. J Biol Chem. 2009;284(7):4626-34.

43. Lo RK, Cheung H, Wong YH. Constitutively active Galpha16 stimulates STAT3 via a C-Src/JAK- and ERK-dependent mechanism. J Biol Chem. 2003:278(52):52154-65

44. Cohen B, Shimizu M, Izrailit J, Ng NF, Buchman Y, Pan JG, et al. Cyclin D1 is a direct target of JAG1-mediated Notch signaling in breast cancer. Breast Cancer Res Treat. 2010;123(1):113-24.
45. Kehn K, Berro R, Alhaj A, Bottazzi ME, Yeh WI, Klase Z, et al. Functional consequences of cyclin D1/BRCA1 interaction in breast cancer cells. Oncogene. 2007;26(35):5060-9.

46. Chaudhary B, Khaled YS, Ammori BJ, Elkord E. Neuropilin 1: function and therapeutic potential in cancer. Cancer Immunol Immunother. 2014;63(2):81-99.

47. Rottenberg S, Jaspers JE, Kersbergen A, van der Burg E, Nygren AO, Zander SA, et al. High sensitivity of BRCA1-deficient mammary tumors to the PARP inhibitor AZD2281 alone and in combination with platinum drugs. Proc Natl Acad Sci U S A. 2008;105(44):17079-84.

48. De Summa S, Pinto R, Sambiasi D, Petriella D, Paradiso V, Paradiso A, et al. BRCAness: a deeper insight into basal-like breast tumors. Ann Oncol. 2013;24 Suppl 8:viii13-21.

49. Lowery AJ, Miller N, Devaney A, McNeill RE, Davoren PA, Lemetre C, et al. MicroRNA signatures predict oestrogen receptor, progesterone receptor and HER2/neu receptor status in breast cancer. Breast Cancer Res. 2009;11(3):R27.

50. Janssen EA, Slewa A, Gudlaugsson E, Jonsdottir K, Skaland I, Soiland H, et al. Biologic profiling of lymph node negative breast cancers by means of microRNA expression. Mod Pathol. 2010;23(12):1567-76.

51. Sandhu R, Rivenbark AG, Mackler RM, Livasy CA, Coleman WB. Dysregulation of microRNA expression drives aberrant DNA hypermethylation in basal-like breast cancer. Int J Oncol. 2014;44(2):563-72.

52. Jonsdottir K, Janssen SR, Da Rosa FC, Gudlaugsson E, Skaland I, Baak JP, et al. Validation of expression patterns for nine miRNAs in 204 lymph-node negative breast cancers. PLoS One. 2012;7(11):e48692.

53. Joosse $\mathrm{SA}$, van Beers $\mathrm{EH}$, Tielen $\mathrm{H}_{\mathrm{H}}$, Horlings $\mathrm{H}$, Peterse $J$, Hoogerbrugge $N$, et al. Prediction of BRCA1-association in hereditary non-BRCA1/2 breast carcinomas with array-CGH. Breast Cancer Res Treat. 2009;116(3):479-89.

54. Joosse SA, Brandwijk KI, Mulder L, Wesseling J, Hannemann J, Nederlof PM. Genomic signature of BRCA1 deficiency in sporadic basal-like breast tumors. Genes Chromosomes Cancer. 2011;50(2):71-81.

55. van Beers EH, van Welsem T, Wessels LF, Li Y, Oldenburg RA, Devilee P, et al. Comparative genomic hybridization profiles in human BRCA1 and BRCA2 breast tumors highlight differential sets of genomic aberrations. Cancer Res. 2005;65(3):822-7.

56. Jonsson G, Naylor TL, Vallon-Christersson J, Staaf J, Huang J, Ward MR, et al. Distinct genomic profiles in hereditary breast tumors identified by arraybased comparative genomic hybridization. Cancer Res. 2005;65(17):7612-21.

\section{Submit your next manuscript to BioMed Central and take full advantage of:}

- Convenient online submission

- Thorough peer review

- No space constraints or color figure charges

- Immediate publication on acceptance

- Inclusion in PubMed, CAS, Scopus and Google Scholar

- Research which is freely available for redistribution 